\title{
Heterogeneous data fusion for three-dimensional gait analysis using wearable MARG sensors
}

\section{Sen Qiu*, Zhelong Wang and Hongyu Zhao}

School of Control Science and Engineering,

Dalian University of Technology,

Dalian, 116024, China

E-mail: qiu@mail.dlut.edu.cn

E-mail: wangzl@dlut.edu.cn

E-mail: zhy.lucy@hotmail.com

*Corresponding author

\section{Huosheng $\mathrm{Hu}$}

Department of Computer Science and Electronic Engineering,

University of Essex,

Essex, Colchester, CO4 3SQ, UK

E-mail: hhu@essex.ac.uk

\begin{abstract}
Gait analysis has become a research highlight. In this paper, we propose a computing method using wearable MARG (magnetic angular rate and gravity sensor arrays) with wireless network, which can calculates absolute and relative orientation and position information of human foot motion during level walking and stair climbing process. Three-dimensional foot orientation and position were estimated by a Kalman based sensor fusion algorithm and validated by ground truth provided by Vicon system. The repeatability of the alignment procedure and the measurement errors were evaluated on healthy subjects. Experimental results demonstrate that the proposed method has a good performance at both motion patterns. No significant drifts exist in the overall results presented in the paper. The measured and estimated information can be transmitted to remote server through internet. Moreover, this method could be applied to other cyclical activity monitoring.
\end{abstract}

Keywords: Gait analysis, Attitude computation, Wireless sensor network, Wearable sensors, MARG.

Biographical notes: Sen Qiu received the B.S.degrees in automation from Dalian University of Technology, Dalian, China, in 2009. where he has been pursuing the Ph.D. degree in control theory and control engineering with the School of Control Science and Engineering since 2011. He had been a visiting researcher with Department of Computer Science and Electronic Engineering, University of Essex, UK from 2013 to 2014. His current research interests include gait analysis, body sensor network, embedded system design and pattern recognition.

Zhelong Wang(M04) received the B.Sc. and M.Sc.degrees in automatic control from the Dalian University of Technology, Dalian, China, in 1996 and 1999, respectively, and the Ph.D. degree in robotics from the University of Durham, Durham, U.K., in 2003. In 2004, he joined the School of Electronic and Information Engineering, Dalian University of Technology, where he is currently a Professor. His research interests include robotics, intelligent control, mechatronics, body sensor networks, and machine learning.

Hongyu Zhao received the B.S. degree in automation from Dalian University of Technology, Dalian, China, in 2007, where she has been pursuing the Ph.D. degree in control theory and control engineering with the School of Control Science and Engineering since 2009. Her current research interests include pedestrian navigation, indoor localization, inertial navigation and positioning, and gait analysis.

Huosheng $\mathrm{Hu}$ (M'94-SM'01) received the M.Sc.degree in industrial automation from the Central South University, Changsha, China, in 1982 and the Ph.D. degree in robotics from the University of Oxford, Oxford, U.K., in 1993. Currently, he is a Professor with the School of Computer Science and Electronic Engineering, University of Essex, Colchester, U.K., leading the Human-Centered Robotics Group. His research interests include behavior-based robotics, human-robot interaction, service robots, embedded systems, data fusion, learning algorithms, mechatronics, and pervasive computing. He has authored around 360 papers in journals, books, and conferences in these areas. 


\section{Introduction}

Gait analysis is an objective qualitative analysis or quantitative evaluation of the human locomotion, such as walking, running, and climbing. Using kinematics concepts and existing knowledge of human anatomy and physiology, kinematic and kinetic data are acquired and analyzed to provide information which describes fundamental gait characteristics and which is ultimately interpreted by the clinicians to form an assessment (Davis et al., 1991). Specifically, gait analysis can be an efficient evaluation and guidance for pedestrian navigation, medical and other commercial applications.

Existing work has employed a number of different methodologies including electromyographic (EMG) signal, optical sensing, acoustic tracker, etc. Nymark et al. (2005) compared the kinematic and EMG gait patterns of able-bodied adults at natural speed in contrast to extremely slow over ground and treadmill walking speeds. Mihradi et al. (2011) presented the development of an optical motion-capture system for 3D gait analysis using two video cameras. The analysis of the participant's head and limbs has been done by using ultrasonic acoustic system (Gilson et al., 2006). The above methods can be effective for certain applications. However, Muscle signal based methods require a specialized medical staff and a dedicated working space, therefore, these methods avoid ambulatory monitoring; Ultrasonic technologies are mature but the complexity and cost are often prohibitive for routine evaluations. Optical based methods have low jitter and stability merits but need expensive graphic signal processing devices, which is not practical in a home based environment. The above described methods are difficult to be applied in daily life usage scenario.

Wireless sensor network (WSN) is widely used to set up monitoring and tracking system in recent years, which offers us new solutions to the aforementioned problems. In literature, Srinivasan and Ranganathan (2013) adopted RFID sensor network for continuously asset monitoring but the relatively high operating costs of RFID limited its application. $\mathrm{Ai}$ et al. (2014) introduced a Wi-Fi fingerprint indoor localization method for smart phone's heterogeneity, however, the major problem for Wi-Fi fingerprinting is that the signal strength detection by different devices is seriously affected, thus deteriorating the localization accuracy to a large extent. Talpur et al. (2015) proposed a novel shared-node IoT network architecture with ubiquitous homomorphic encryption for healthcare monitoring using non-ubiquitous technologies. Meanwhile, the widely used IMU (Inertial measurement unit) which consists of an accelerometer and a gyroscope has proven to be applicable for measurement of position and orientation in a short period (Yun et al., 2007), (Meng et al., 2014). The benefits of using a wearable IMU include the following: they are of small size, have low cost, testing is not restricted to a settled environment, and they can be easily integrated with existing platforms for body sensor networks (Jiang et al., 2011). Three dimensional position and orientation can be calculated by integrating the accelerometer and gyroscope output respectively from initial conditions determined by an accelerometer. As reported in previous studies, Wang et al. (2012) applied inertial sensors in walking pattern classification and walking distance estimation, Bamberg et al. (2008) developed a shoe-integrated gait analysis system using IMUs and force sensors. However, IMU can only measure an attitude relative to the gravity direction, which means signal of the accelerometer is not affected by a rotation around the vertical direction, hence additional information is required to compensate for the heading drift (Sabatini, 2012). MARG systems, i.e. magnetic angular rate and gravity sensor arrays, are good solutions to provide a complete measurement of orientation relative to the direction of gravity and the earth magnetic field.

There are two prevalent problems associated with MARG application, the initial errors caused by sensor misplacement and drift errors during integral operation, especially in a long period of time. To address the first problem, one can adopt an alignment procedure at the beginning of each data collection. On the other hand, the accelerometer can provide drift-free inclination estimates by sensing the gravity field and eliminating drifts by continuous correction of the orientation obtained using rate sensor data. Kalman filter is often used to fuse the sensor data (Qiu et al., 2014). Finally, the foot position, orientation and gait phase information could be obtained and evaluated.

The organization of this paper is as follows. A brief system overview is presented in section 2 . The orientation and position estimation algorithm are presented in section 3. Subsequently, the experimental results and the algorithm validation are given in section 4. Comparisons with literature are presented in section 5. Finally, we draw our conclusion and future research work in section 6 .

\section{System overview}

\subsection{The experiment platform}

In our previous study(Wang et al., 2013), a gait analysis system was designed in our lab but the sensor precision was relatively low and the alignment errors between the sensor chip and the plastic housing were not well controlled, so we simplified the three-dimensional motion as two-dimensional, i.e. we merely considered the sensor data in the sagittal plane, in this case, measurement errors were reduced but the abductionadduction and internal-external rotation of foot were ignored likewise. In this study, we adopted an off-theshelf miniature MARG sensor unit which has better performance, as shown in TABLE 1. The accelerometer, gyroscope and magnetometers are all composed of sensor triplets, with their sensitivity axes being mutually 
Figure 1 Sensor attachment and Vicon marker placement

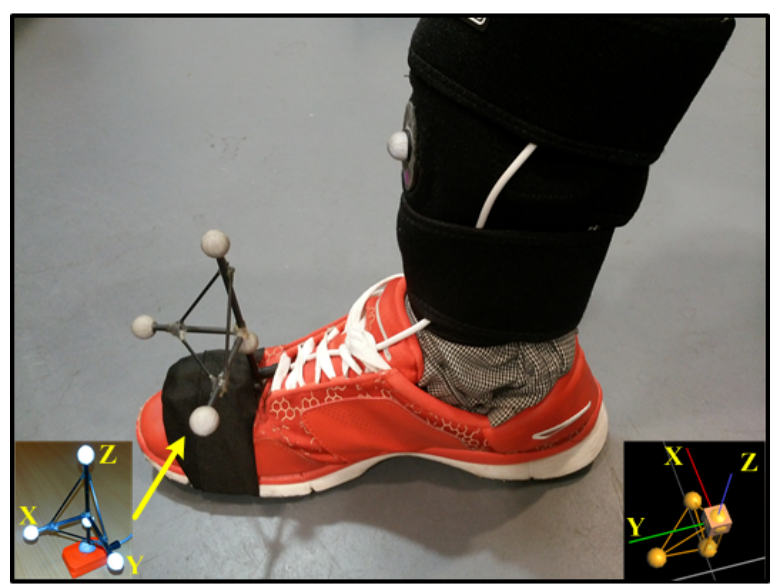

orthogonal. The output of three-dimensional linear acceleration, three-dimensional gyroscope and threedimensional magnetometer all have orthogonal $\mathrm{X}, \mathrm{Y}$ and $\mathrm{Z}$ axis readings within $0.1^{\circ}$, and the sensor errors are minimized accordingly. In regard with the data fusion, we adopted the Kalman filter to fuse different types of sensor data, obtaining complete three-dimensional orientation and position tracking of foot during walking trials.

Table 1 MARG PERFORMANCE SPECIFICATION

\begin{tabular}{cccc}
\hline \hline Unit & Accelerometer & Gyroscope & Magnetometer \\
\hline Dimensions & 3 axes & 3 axes & 3 axes \\
Dynamic Range & $\pm 50 \mathrm{~m} / \mathrm{s}^{2}$ & $\pm 1200 \mathrm{deg} / \mathrm{s}$ & $\pm 750 \mathrm{mGauss}$ \\
Bandwidth $(\mathrm{Hz})$ & 30 & 40 & 10 \\
Bias stability (unit $1 \sigma)$ & 0.02 & 1 & 0.1 \\
Noise density $(1 / \sqrt{\mathrm{Hz}})$ & 0.02 & 0.05 & $0.5(1 \sigma)$ \\
\hline \hline
\end{tabular}

When using wearable sensor to track human movement, it is always better to position the measurement device at a steady position. In this way, the tracking sensor is snugly strapped to the subjects' instep with hook-and-loop fastener bands. In this case, the position of the sensor is away from the joints and avoids poor rotation estimation in consequence. The sensor is placed in such a way that the top of the sensor faces upwards when the foot is naturally flat. Figure 1 shows the sensor attachment and Vicon marker placement. Sensor data is measured with a sampling frequency of $100 \mathrm{~Hz}$ and transmitted to a laptop via Blue-tooth network, which is done by a digital data box called XBus placed on the waist. This wireless feature enables the subject to carry out the experiment freely. For each layer of the stair, the height is $0.16 \mathrm{~m}$ and the width is $0.3 \mathrm{~m}$.

\subsection{Optical reference system}

To verify the effectiveness of the wearable sensor based algorithm, comparisons were made between the quantitative results of the sensor system and the measurements of a visual marker based Vicon system.
In our lab, Vicon system is placed in the Robot Arena above the 100 square metre powered floor, consisting of eight MX F40 cameras connected to an MX ultranet server and Nexus software. The information of optical markers attached to the subject can be logged at 100 $\mathrm{Hz}$ (the same as the sampling frequency of wearable sensors) and then processed to compute the orientation of the measurement site. In order to ensure the measurements of orientation and position in the camera coordinate frame to be comparable to the estimation algorithm in the ground frame, an initial calibration procedure is always performed where a five markers mini calibration frame is used. This step is required as the cameras may shift over time. After the attachment, each marker can be seen by at least six cameras, which is enough for the system evaluation. Then Vicon system calculates the three-dimensional position and orientation using advanced algorithms. The implementation of such algorithms is beyond the scope of this study. The reference model consists of four tiny reflective balls was put on the sensor which was attached on the foot. The coordinate system of the reference model is shown in the left bottom of Figure 1 and the reference model in Vicon software is illustrated in the right bottom. In our study, we define X-axis as "forward" direction, Y-axis as "left" direction and Z-axis as the "upward" direction, respectively.

\section{Methodology}

\subsection{Representation of orientation and position}

Gait motion can be described as rigid body motion based on its physiological property. There are three commonly used methods to illustrate the rigid body rotation, i.e. rotation matrices, Euler angle and quaternion. Methods based on Euler angles are unable to track orientation when a rigid body is in a vertical orientation, a serious limitation when tracking movements of human limb segments (Bachmann and Zyda, 2000).The algorithm in this paper employs a quaternion representation of orientation to describe the coupled nature of orientations in three dimensions.

Quaternion requires less calculation time than rotation matrices and is not subject to the existed singularities problem associated with an Euler angle representation. The quaternion is represented as formula (1). And the notation system of leading superscripts and subscripts is used to identify which coordinate system a quantity is described. For instance, ${ }_{S}^{R} q$ describes the orientation of coordinate system $S$ relative to $R$. On the other hand, trailing superscripts are used for indicating the inverse or transpose of a matrix, e.g., $q^{-1}$. For a united quaternion, ${ }_{S}^{R} q^{-1}$ equals ${ }_{R}^{S} q$.

$$
q=\left(q_{0}, q_{1} i, q_{2} j, q_{3} k\right)
$$

where $i, j$, and $k$ denote the standard orthonormal basis for $3 \mathrm{D}$ space. To simplify the writing mode, let $q=$ 
$\left(q_{0}, q_{1}, q_{2}, q_{3}\right)$, then the quaternion conjugate of $q$ is $q^{*}=$ $\left(q_{0},-q_{1},-q_{2},-q_{3}\right)$. The norm of a quaternion is called the magnitude of the quaternion,

$$
N(q)=\sqrt{q_{0}^{2}+q_{1}^{2}+q_{2}^{2}+q_{3}^{2}}
$$

and a quaternion with a unity norm is called unit quaternion.

$$
q_{u n i t}=q / N(q)
$$

Since rotation is given as angular rate by gyroscope. A unit quaternion vector can be interpreted to represent a rotation about a unit vector $\mathrm{N}$ through an angle $\alpha$,

$$
q=\left(q_{0}, q_{1}, q_{2}, q_{3}\right)=\left(\cos \frac{\alpha}{2}, N \sin \frac{\alpha}{2}\right)
$$

There are no singularities and only two trigonometric functions involved. The rigid body angular motion obeys the vector differential equation

$$
\dot{q}(t)=\Omega[\omega \times] * q(t)
$$

where

$$
\Omega[\omega \times]=\frac{1}{2} \Omega\left[\begin{array}{cccc}
0 & -\omega_{z} & \omega_{y} & \omega_{x} \\
\omega_{z} & 0 & -\omega_{x} & \omega_{y} \\
-\omega_{y} & \omega_{x} & 0 & \omega_{z} \\
-\omega_{x} & -\omega_{y} & -\omega_{z} & 0
\end{array}\right]
$$

where $\omega_{x}, \omega_{y}$ and $\omega_{z}$ represent the output of gyroscope. In this way the relationship is established between angular rate and quaternion. The transformation between the representations, relative to $B$ and $G$. A $3 \times 1$ column vector $V(k)$, whose components are generally functions of time, is expressed as follows:

$$
\begin{aligned}
& { }_{B}^{G} R=\left[\begin{array}{l}
1-2\left(q_{2}^{2}+q_{3}^{2}\right) 2\left(q_{1} q_{2}-q_{0} q_{3}\right) 2\left(q_{1} q_{3}+q_{0} q_{2}\right) \\
2\left(q_{1} q_{2}+q_{0} q_{3}\right) 1-2\left(q_{1}^{2}+q_{3}^{2}\right) 2\left(q_{2} q_{3}-q_{0} q_{1}\right) \\
2\left(q_{1} q_{3}-q_{0} q_{2}\right) 2\left(q_{2} q_{3}+q_{0} q_{1}\right) 1-2\left(q_{1}^{2}+q_{2}^{2}\right)
\end{array}\right] \\
& { }^{G} V(k)={ }_{B}^{G} q(k)^{B} V(k){ }_{B}^{G} q^{*}(k)={ }_{B}^{G} R^{B} V(k)
\end{aligned}
$$

where $\mathrm{k}$ is sample sequence and $\mathrm{T}$ is the sampling time (0.01 second in this paper). Considering that it is supposed to evaluate the subject motion in the ground reference coordinate, as defined above ${ }_{B}^{G} q$ rotates a vector $V$ in the body fixed frame B to the ground reference coordinate system $\mathrm{G}$. The orientation of a rigid body in space is determined when the axis orientation of a coordinate frame attached to the body (the body frame) is specified with respect to the ground frame G. ${ }_{G}^{B} q^{*}(k)$ is the complex conjugate of ${ }_{G}^{B} q(k)$.

The velocity and the position in the ground reference coordinate, ${ }^{G} v$ and ${ }^{G} p$ can be estimated by integrating the acceleration. Consider a rigid body in space and define a coordinate system, the body frame is attached to the body. The linear three-dimensional accelerometers measure all accelerations, including the acceleration due to gravity. This is inherent to all accelerometers. Therefore, gravity must first be subtracted, as shown below. In a discrete time domain, position is calculated as follows:

$$
\left\{\begin{array}{l}
{ }^{G} a(k)={ }_{B}^{G} R\left({ }^{B} a(k)-g\right) \\
{ }^{G} v(k)={ }^{G} v(k-1)+\frac{T}{2}\left({ }^{G} a(k)+{ }^{G} a(k-1)\right) \\
{ }^{G} p(k)={ }^{G} p(k-1)+{ }^{G} v(k) * T+\frac{T^{2}}{2}\left(\frac{{ }^{G} a(k)+{ }^{G} a(k-1)}{2}\right)
\end{array}\right.
$$

where ${ }^{G} a(k)$ is acceleration, ${ }^{G} v(k)$ the velocity and ${ }^{G} p(k)$ the position presented in the ground frame.

\subsection{Quaternion convergence and kalman filter design}

Considering the inevitable sources of errors including misalignment between couples of axes in each sensor and inherent error of the sensors, in reality there is no existence of an ideal quaternion which exactly converts the body frame to the ground frame. One solution is to set up the error function, determining the optimal quaternion such that the errors (E) are minimized. A useful method is the iterative splitting method which ensures a consistent approximation in each step (Farago and Geiser, 2007).

$$
\begin{aligned}
& E=\varepsilon^{T} \varepsilon=\left({ }^{G} V_{1}-D^{B} V_{0}\right)^{T}\left({ }^{G} V_{1}-D^{B} V_{0}\right) \\
& D=\left[\begin{array}{cc}
R & 0 \\
0 & R
\end{array}\right]
\end{aligned}
$$

where ${ }^{G} V_{1}$ is a $6 \times 1$ dimension vector including the values of gravity and magnetic field represented in the ground frame, ${ }^{B} V_{0}$ is the measurements of gravity and magnetic field in the body frame with the same dimension.

In view of that ${ }^{B} V_{0}$ is measured and ${ }^{G} V_{1}$ is known, the error actually depends on the four components of the quaternion. The objective function is to determine the quaternion that yield the minimum error through iterative operation. A Gauss-Newton algorithm is adopted to use the first derivative of the error function. It is defined as:

$$
\hat{q}_{k+1}=\hat{q}_{k}-\left[J^{T}\left(\hat{q}_{k}\right) J\left(\hat{q}_{k-1}\right)\right]^{-1} J^{T}\left(\hat{q}_{k}\right) \varepsilon\left(\hat{q}_{k}\right)
$$

where vector q stands for the four components of the quaternion and $\mathrm{J}$ is the Jacobian matrix given as:

$$
J=-\left[\left(\frac{\partial D}{\partial q_{0}}{ }^{B} V_{0}\right)\left(\frac{\partial D}{\partial q_{1}}{ }^{B} V_{0}\right)\left(\frac{\partial D}{\partial q_{2}}{ }^{B} V_{0}\right)\left(\frac{\partial D}{\partial q_{3}}{ }^{B} V_{0}\right)\right]
$$

The experimental results show that the algorithm is convergent and converges in six steps in most cases, further details are provided later in this article.

Kalman filter is an algorithm using a series of measurements observed over time, containing noises and other inaccuracies, and produces estimates of unknown variables that tend to be more precise than those based on a single measurement alone. The algorithm works in a two-step process. In the prediction step, the Kalman filter produces estimates of the current state variables, along with their uncertainties. Once the outcome of the next measurement is observed, these estimates are 
Figure 2 Flow-chart of integration algorithm

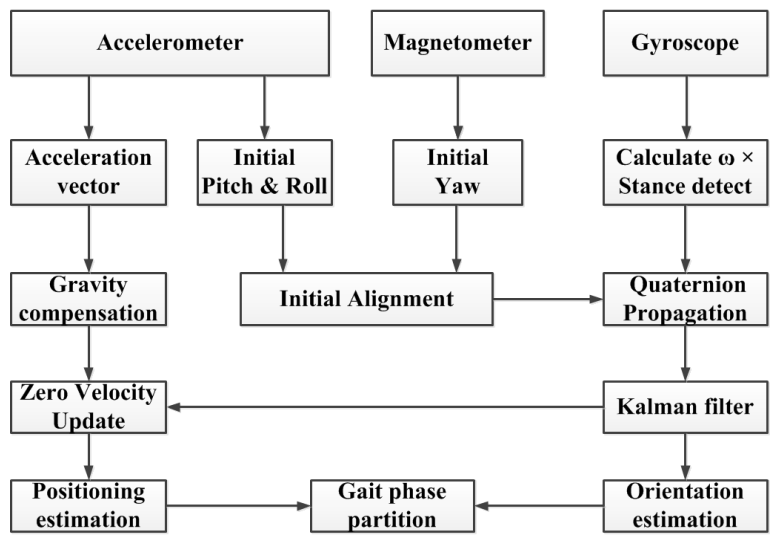

updated using a weighted average, more weight will be given to estimate with higher certainty. Owing to the recursive nature, it is feasible to run the algorithm in real time using merely the current input measurements and the previous state and the corresponding uncertainty matrix. The Kalman equations are summarized as follows. The state equation is represented by measuring error of gyroscope $\Delta \theta$ and bias offset of the gyroscope outputs $\triangle \delta$ as follows:

$$
\left[\begin{array}{ll}
\Delta \theta_{k+1} \\
\Delta & \delta_{k+1}
\end{array}\right]=\left[\begin{array}{ll}
1 & T \\
0 & 1
\end{array}\right]\left[\begin{array}{ll}
\Delta & \theta_{k} \\
\Delta & \delta_{k}
\end{array}\right]+\left[\begin{array}{c}
T \\
1
\end{array}\right] \mu
$$

where $\mu$ is the measurement error with gyroscope. Observation signal is the difference of angles obtained from the gyroscope and the accelerometer $\Delta b$, which is given by

$$
\triangle \rho_{k}=\left[\begin{array}{ll}
1 & 0
\end{array}\right]\left[\begin{array}{c}
\Delta \theta_{k} \\
\triangle \delta_{k}
\end{array}\right]+\sigma
$$

where $\sigma$ is the measurement error with accelerometer. Kalman filter repeats (16) and (17) according to the following state space model:

$$
\begin{aligned}
& {\left[\begin{array}{c}
\Delta \hat{\theta}_{k} \\
\Delta \hat{\delta}_{k}
\end{array}\right]=\left[\begin{array}{l}
\Delta \hat{\theta}_{k}^{-} \\
\Delta \hat{\delta}_{k}^{-}
\end{array}\right]+\left[K_{1} K_{2}\right]\left(\Delta \rho_{k}-\Delta \hat{\theta}_{k}\right)} \\
& {\left[\begin{array}{l}
\Delta \hat{\theta}_{k+1}^{-} \\
\Delta \hat{\delta}_{k+1}^{-}
\end{array}\right]=\left[\begin{array}{ll}
1 & T \\
0 & 1
\end{array}\right]\left[\begin{array}{l}
\Delta \hat{\theta}_{k} \\
\Delta \hat{\delta}_{k}
\end{array}\right]}
\end{aligned}
$$

where $K_{1}$ and $K_{2}$ represent Kalman gain for $\Delta \theta$ and $\Delta \delta$, $\Delta \hat{\theta}$ and $\Delta \hat{\theta}^{-}$represent estimated value and predicted value for $\Delta \theta$, respectively. For the initial condition, $\Delta \hat{\theta}^{-}$ is set as 0 , and $\Delta \hat{\delta}^{-}$is set as $\Delta \hat{\delta}$ at the last measurement. The Kalman filter is applied repeatedly until its output converged. The flow chart of the proposed method is illustrated in Figure 2.

As shown above, acceleration and angular velocity are fused by kalman filter after initial alignment. The Kalman filter calculates $\Delta \theta$ and $\Delta \delta$ by using the angle difference $\Delta \rho$. In the system, Kalman gain increases as the noise ratio (the covariance of observation noise and process noise) decreases and vice versa. Specifically, large value of Kalman gain for small noise ratio
Table 2 QUATERNION CONVERGENCE

\begin{tabular}{ccccc}
\hline \hline Sample & $q_{0}$ & $q_{1}$ & $q_{2}$ & $q_{3}$ \\
\hline 1 & 0.99989 & 0.00246 & 0.00428 & 0.01157 \\
2 & 0.99997 & 0.00195 & 0.00335 & 0.01237 \\
3 & 0.99996 & 0.00199 & 0.00296 & 0.00296 \\
4 & 0.99998 & 0.00181 & 0.00319 & 0.01156 \\
5 & 0.99998 & 0.00178 & 0.00247 & 0.01097 \\
6 & 0.99998 & 0.00172 & 0.00172 & 0.01095 \\
\hline \hline
\end{tabular}

means that estimation results become highly dependent on acceleration, while small Kalman gain for large noise ratio indicates that calculation results are more dependent on angular velocity. The noise ratio values are determined by trial and error method.

TABLE 2 shows the elements of the quaternion for the first six samples. The initial quaternion is set to $(0.5,0.5,0.5,0.5)$. The practical position of the sensor after initial alignment is represented by the quaternion $(1,0,0,0)$ in the reference frame. The quaternion estimation converged to the practical position in a couple of iterations.

\subsection{Initial state estimation and alignment}

It is common that the sensor axes are not exactly aligned with the axes of the subject of which the orientation has to be recorded. But what we need is to output the inertial data in a body fixed frame other than a sensor fixed frame. Otherwise there will be large errors. In this study an initial alignment method similar to bore sighting was used. When performing an initial alignment, the new ground reference $\mathrm{X}$ coordinate is chose by the forward direction of the subject. $\mathrm{X}$-axis points in the direction of the sensor while keeping the ground Z-axis pointing upwards as shown in Figure 3.

The subjects were required to stand still for a few seconds before performing the experiments, in this way the gravity in condition of zero linear accelerometer can be estimated, in the same time, the earth magnetic field in condition of null yaw can be evaluated. The horizontal component is deducted so that the initial yaw equals zero after the initial alignment process. After the alignment, the initial quaternion $q_{0}$ should be close to $(1,0,0,0)$. Then the inertial data is expressed in the body frame whose orientation is supposed to be recorded. As discussed above, gravity offers us a reference vector $[0,0, g]^{T}$ to calculate the initial roll and pitch using formula (18) and (19).

$$
\begin{aligned}
& \theta_{\text {roll }}=\arctan \left(A c c_{y} / A c c_{x}\right) \\
& \theta_{\text {pitch }}=-\arcsin \left(A c c_{z} / g\right)
\end{aligned}
$$

However, accelerometer is not able to sense the rotation in the $\mathrm{X}-\mathrm{O}-\mathrm{Y}$ plane. In this case earth magnetism provides the other reference vector $\left[m_{x}, m_{y}, m_{z}\right]^{T}$. Unlike gravity which is vertically 
Figure 3 The initial axis alignment

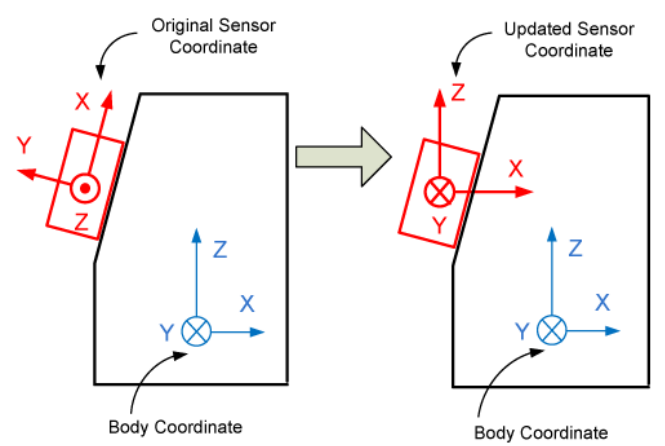

Figure 4 Heading reset by magnetometer

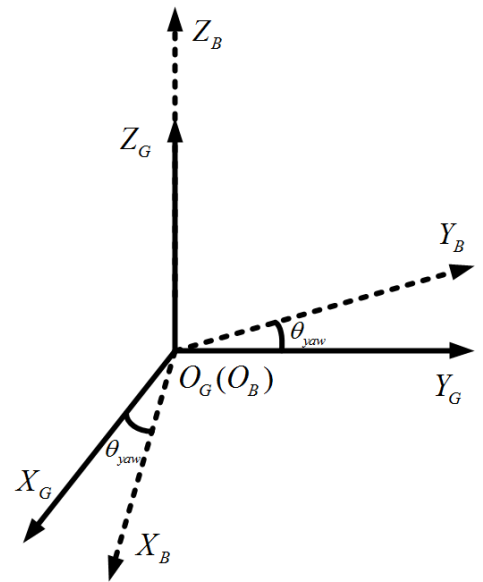

downward, there is an angle between earth magnetism vector and the $\mathrm{X}-\mathrm{O}-\mathrm{Y}$ plane. If we adjust the orientation of the sensor to north direction, the vector turn to $\left[m_{x}, 0, m_{z}\right]^{T}$. In this research gravity vector is firstly used to rotate the body frame to the same X-O-Y plane of ground frame then the magnetometer is adopted to calculate the difference of yaw. After the correction of the magnetometer, initial $\theta_{\text {yaw }}$ is determined, as illustrated in Figure 4 and formula (20),

$$
\theta_{\text {yaw }}=\arctan \left(\operatorname{Mag}_{y}, \operatorname{Mag}_{x}\right),
$$

where $M_{a} g_{x}$ and $M_{a g}$ are magnetic sensor measurements of $\mathrm{X}$-axis and $\mathrm{Y}$-axis, respectively.

\section{Experiment setup and experimental results}

Walking is the most important function of human gait. Therefore, most of the researches are involved with walking evaluation. In others studies participants data is collected during walking upstairs and downstairs (Lamontagne et al., 2011), In our study, experiments were applied in two ways, the normal way of level walking on the specified ground and a controlled way of going upstairs. Six subjects (three males and three females with a mean age of $30 \pm 6$ years, a mean weight $63 \pm 17 \mathrm{~kg}$ and a mean height of $1.71 \pm 0.13$ meters) were analyzed during walking at the specified
Figure 5 Filtered data from MARG sensors of level walking

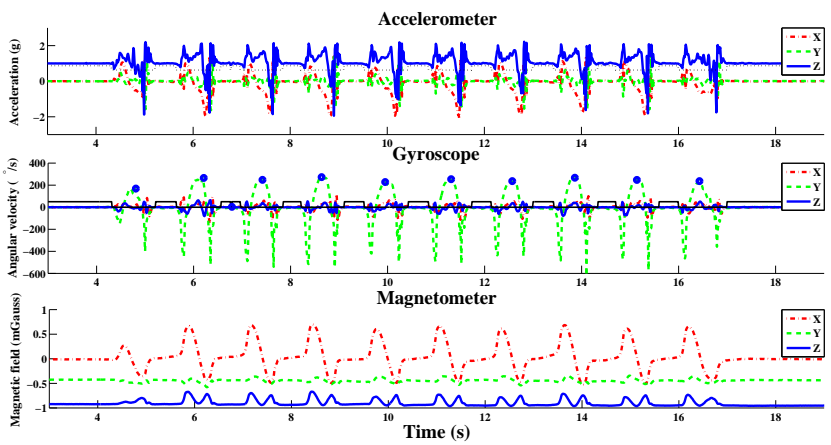

site. Neither of them has a history of lower limb pathologic conditions. Stair climbing capacity has perioperative prognostic medical importance. It may predict postoperative recovery and complications for stroke rehabilitation. Moreover, the $\mathrm{Z}$ axis component is more distinct than flat level walking trials. We carried out the experiment in the second scenario where subjects were instructed to climb stairs. Unlike level walking when each individual performs his or her own unique pace with different walking speed, each step of the stair climbing is restricted by the fixed stair height and inclination. This offers us a reference on condition that other precise reference system such as Vicon is not available in the stairwell. We asked our volunteers to perform both experimental patterns.

\subsection{Orientation and position estimation}

Raw data from the MARG system is filtered by both of high pass $(0.001 \mathrm{~Hz})$ and low pass filter $(5 \mathrm{~Hz})$. Figure 5 describes the filtered sensor data of level walking. We have developed a walking distance estimation algorithm based on the filtered sensor data. Step numbers can be easily calculated using gyroscope data, with each step marked with a blue peak circle on the Y-axis gyroscope curve. Stance stages, when the corresponding foot is firmly standing on the ground, are distinguished with a threshold algorithm using acceleration data. As shown with dot line under the curve of Z-axis accelerometer.

According to the characteristics of zero velocity of the stance phase (Skog et al., 2010), we filtered out the drift of the acceleration signal, in this case the error from the previous step will not propagated to the current step. Then we estimated the walking distance by taking double integrals of the filtered acceleration combine with angular information from gyroscope. In the first scenario, the subjects were asked to walk on the level floor of robot arena. Figure 6 illustrates the 3D trajectory of level walking in robot arena. It turned out that the corresponding subject walked straightly from the origin for ten steps and moved forward with 10.24 meters and 0.33 meters in the left direction. During the stairs climbing experiment, the subjects were asked to climb two flights of 10 steps with normal speed at the Network 
Figure 6 Three-dimensional trajectory of level walking

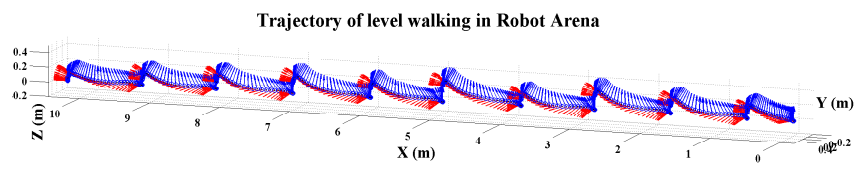

Figure 7 Three-dimensional trajectory of stairs climbing

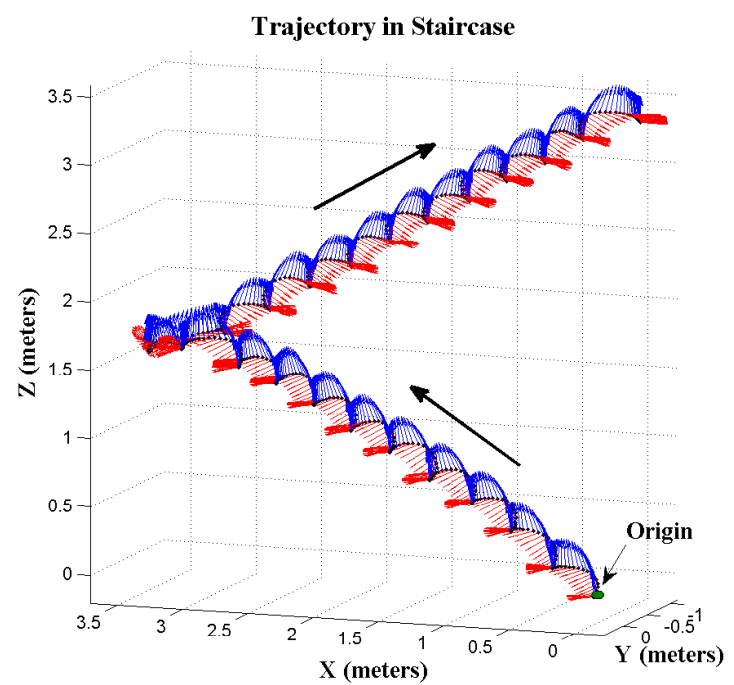

Centre of University of Essex. The stair is a standard one with each layer $16 \mathrm{~cm}$ height and $30 \mathrm{~cm}$ width. Figure 7 illustrates one subject's entire stair climbing trajectory in three dimensions.

\subsection{Effectiveness and repeatability evaluation of proposed method}

It is always important to judge the effectiveness of a method. In our study, we have a tool to gain ground truth when the subjects performed the level walking experiments. One subject was asked to perform the level straight line walking for five steps (not more than 5.5 meters) due to the limited area defined by Vicon system. Since there is no significant displacement on Z-axis during level walking on the whole, two-dimension foot position calculated by proposed method was compared with the position information from Vicon system, as shown in Figure 8. There exist some significant differece errors especially when the subject performed heel striking motion, which may due to the signal delay of wearable sensors. However the maximum errors are not more than $0.02 \mathrm{~m}$. Overall, the comparative results are satisfactory. To validate if the method works well across different subjects, six subjects are instructed to perform level walking along the $5 \times 4$ meters rectangular route for one lap at self-administered speeds. The experimental results of walking distance are shown in TABLE 3 .
Figure 8 Difference errors of two-dimensional position estimation between proposed method and Vicon results

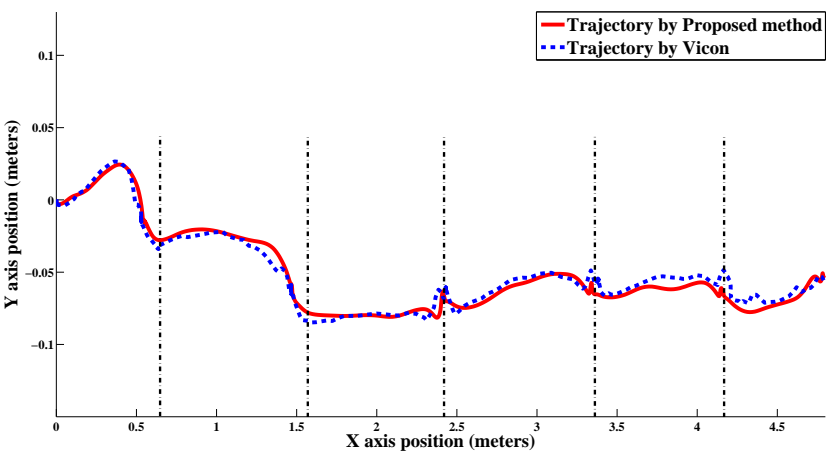

Figure 9 (a)Pitch estimation of one gait cycle by proposed method, Xsens and Vicon system (b)Difference errors of pitch estimation of proposed method and Xsens results

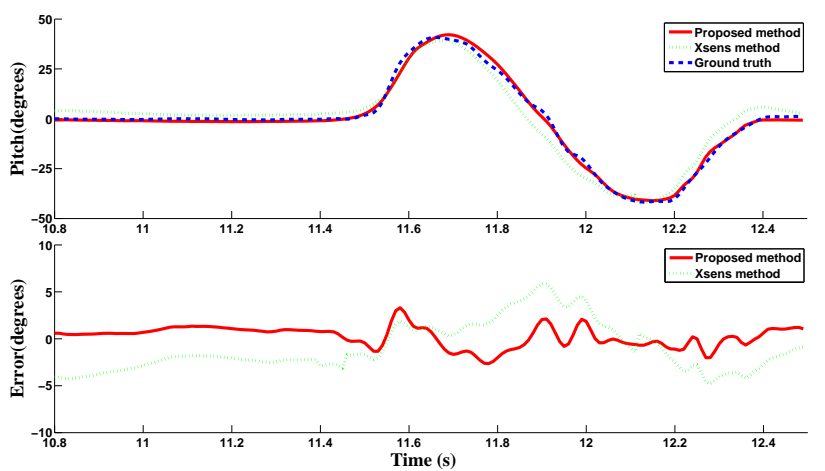

Table 3 THE PERFORMANCE OF DISTANCE ESTIMATION METHOD ACROSS DIFFERENT SUBJECTS

\begin{tabular}{cccc}
\hline \hline $\begin{array}{c}\text { Experimenter } \\
\text { (True value) }\end{array}$ & $\begin{array}{c}\text { Step count } \\
\text { (True value) }\end{array}$ & $\begin{array}{c}\text { Distance }(\mathrm{m}) \\
\text { (True value) }\end{array}$ & $\begin{array}{c}\text { Distance } \\
\text { \%Error }\end{array}$ \\
\hline Subject $\sharp 1$ & $28(28)$ & $19.768(19.918)$ & -0.76 \\
Subject $\sharp 2$ & $25(25)$ & $20.624(20.676)$ & -0.25 \\
Subject $\sharp 3$ & $30(30)$ & $22.211(22.169)$ & 0.19 \\
Subject $\sharp 4$ & $27(27)$ & $20.936(21.106)$ & -0.81 \\
Subject $\sharp 5$ & $26(26)$ & $21.353(21.436)$ & -0.39 \\
Subject $\sharp 6$ & $29(29)$ & $22.139(22.267)$ & -0.58 \\
\hline \hline
\end{tabular}

The method can achieve an average distance error of $0.50 \%$ for level walking and an average height error of $1.7 \%$ for stair climbing in an indoor environment. With respect to the effectiveness of orientation estamation, Figure 9 illustrates the principal component, i.e., pitch estimation during one gait cycle by proposed method, Vicon and Xsens software respectively. Obviously the proposed method gain a better result (errors less than $3^{\circ}$ ) than the typical kalman filter used by Xsens (errors reach $5^{\circ}$ ), which is susceptible to magnetic disturbance. 
Figure 10 Three-dimensional position estimation of stairs climbing

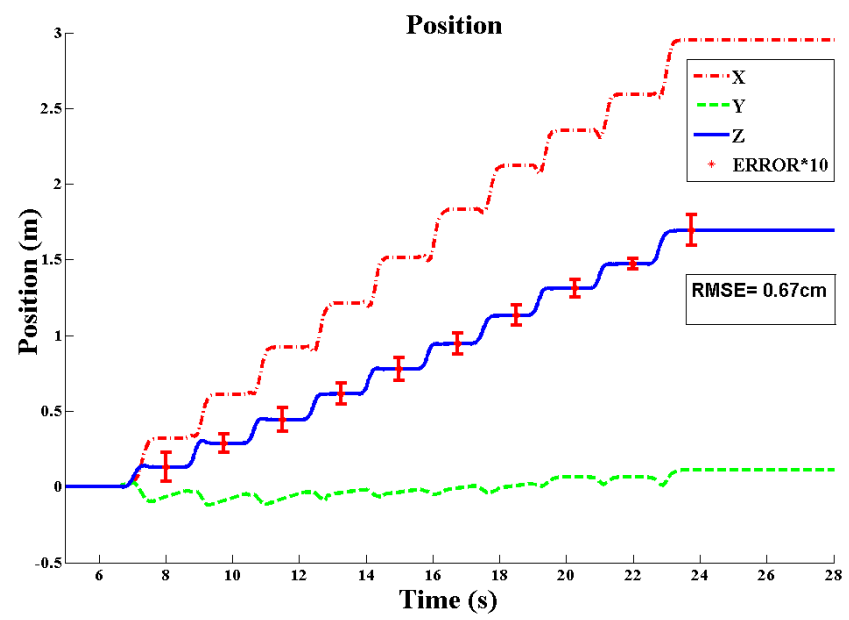

Since Vicon system is not available in the stairwell, we evaluated the ten steps of stairs climbing motion using the fix step height of each layer as ground truth. Figure 10 describes the three dimension upstairs walking distance. After climbing the 10 steps stair by one subject, the calculated position on three dimension are: 295.09 $\mathrm{cm}$ (X axis), $11.09 \mathrm{~cm}$ (Y axis) and $161.92 \mathrm{~cm}$ ( $\mathrm{Z}$ axis). In order to make the errors more distinct, they are multiplied by 10 . In terms of step height, the RMSE of ten steps is $0.67 \mathrm{~cm}$ validated by ground truth.

In some cases, there could be slide between the bandage and the foot during the trial, which is a deterioration of algorithm accuracy. On account of this latent problem, the repeatability of the proposed method was evaluated. In the validation procedure the same subject was asked to perform upstairs walking trial for five times while the sensor was detached and then replaced between each test. The errors were determined for each trial and the mean and standard deviation were calculated based on the obtained ground truth. It is hard to record the drop point of each step during the trial, but the height is determinated by the layer height. Hence the RMSE (root mean square error) of vertical displacement is considered as the repeatability error due to the sensor attachment. The whole results are summarized in TABLE 4, which outlines the minimum, maximum, mean value, expectation value (EX), and root mean square error (RMSE) of five upstairs walking trials. The biggest errors appear in the first and last step, which is predictable because of the initial acceleration and the deceleration at the end. The average RMSE is $0.28 \mathrm{~cm}$, which is less than $2 \%$ of the EX $(16 \mathrm{~cm})$.

\subsection{Gait phase partition}

As proposed in Cavallo et al. (2005), a gait cycle can be divided into swing and stance phase. The swing phase is the period of time when the foot is not in contact with the ground while the period of foot contacting with ground is defined as stance phase. In the following study
Table 4 REPEATABILITY OF POSITION ESTIMATION

\begin{tabular}{cccccc}
\hline \hline Statistics $(\mathrm{cm})$ & Min & Max & Mean & EX & RMSE \\
\hline Trial 1 & 15.64 & 16.54 & 16.17 & 16 & 0.37 \\
Trial 2 & 15.48 & 16.23 & 15.81 & 16 & 0.15 \\
Trial 3 & 15.79 & 16.32 & 15.86 & 16 & 0.26 \\
Trial 4 & 15.45 & 16.05 & 15.73 & 16 & 0.41 \\
Trial 5 & 15.84 & 16.41 & 16.19 & 16 & 0.23 \\
Mean value & 15.64 & 16.31 & 15.95 & 16 & 0.28 \\
\hline \hline
\end{tabular}

Figure 11 Gait phase partition using sensor data

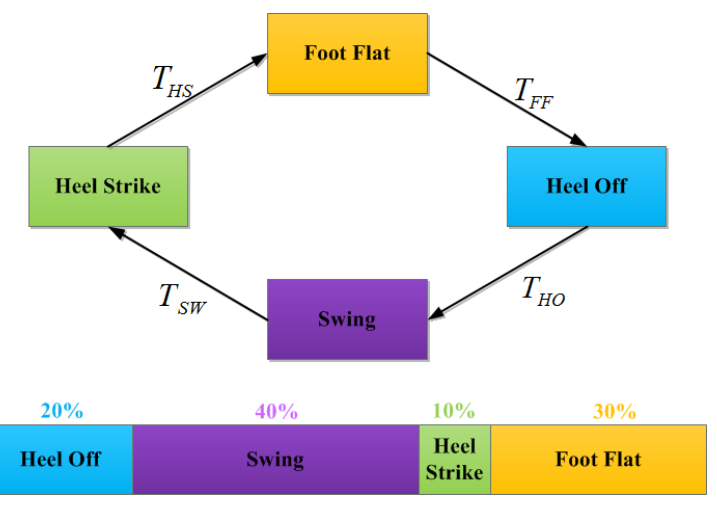

of (Wang et al., 2015), stance phase is divided into heeloff, heel-strike and foot-flat specifically. In this way, a gait cycle can be divided into heel-off, swing, heel-strike and foot-flat, which can be described by Figure 11 .

Figure 12 False stance detection on account of sensor data fluctuation

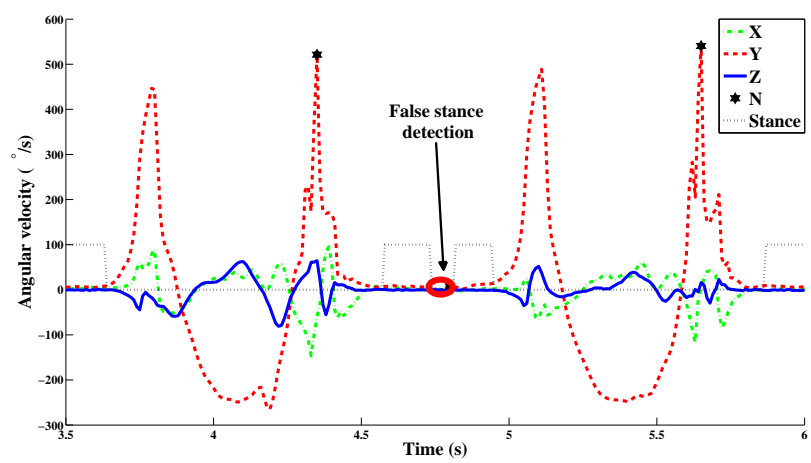

Using the gait phase partition method in our previous research (Wang et al., 2013), the mean value and standard deviation of gait parameters, including stride length(L), heel-off(HO), swing(SW), heel-strike(HS), foot-flat $(\mathrm{FF})$, stride time(T) are presented in TABLE 5 based on the accurate estimation of $3 \mathrm{D}$ foot orientation and position. Experimental results show that men perform larger steps and longer stride length than women. Obviously there is a positive correlation between the stature of the subjects and their stride length. By calculating the percentage of each walking phase in the whole gait cycle, the average values are as follows: HO (28\%), SW (34\%), HS (12\%), and FF (26\%). And false 
Table 5 GAIT PHASE PARTITION

\begin{tabular}{ccccc}
\hline \hline Subjects & $\begin{array}{c}\text { Female } \\
1.62 \mathrm{~m}, 47 \mathrm{~kg}\end{array}$ & $\begin{array}{c}\text { Female } \\
1.66 \mathrm{~m}, 52 \mathrm{~kg}\end{array}$ & $\begin{array}{c}\text { Male } \\
1.72 \mathrm{~m}, 68 \mathrm{~kg}\end{array}$ & $\begin{array}{c}\text { Male } \\
1.78 \mathrm{~m}, 75 \mathrm{~kg}\end{array}$ \\
\hline $\mathrm{SL}(\mathrm{m})$ & $1.069 \pm 0.097$ & $1.124 \pm 0.117$ & $1.213 \pm 0.134$ & $1.300 \pm 0.161$ \\
$\mathrm{TT}(\mathrm{s})$ & $1.271 \pm 0.094$ & $1.209 \pm 0.034$ & $1.191 \pm 0.128$ & $1.225 \pm 0.096$ \\
$\mathrm{HO}(\mathrm{s})$ & $0.282 \pm 0.042$ & $0.276 \pm 0.024$ & $0.337 \pm 0.030$ & $0.276 \pm 0.033$ \\
$\mathrm{SW}(\mathrm{s})$ & $0.432 \pm 0.025$ & $0.422 \pm 0.018$ & $0.408 \pm 0.054$ & $0.412 \pm 0.041$ \\
$\mathrm{HS}(\mathrm{s})$ & $0.352 \pm 0.043$ & $0.209 \pm 0.032$ & $0.150 \pm 0.015$ & $0.184 \pm 0.016$ \\
$\mathrm{FF}(\mathrm{s})$ & $0.305 \pm 0.045$ & $0.302 \pm 0.030$ & $0.296 \pm 0.043$ & $0.353 \pm 0.049$ \\
\hline \hline
\end{tabular}

stance detection on account of sensor data fluctuation as shown in Figure 12 have been avoided. The results are basically similar to the study of Godha and Lachapelle (Godha and Lachapelle, 2008), however, the percentage of heel-off is significantly larger than the study of Godha, which is about $20.5 \%$. The differences may due to sensor attachment and the individual differences, hence the gait phase partition by proposed method is reasonable on the whole.

\section{Comparison with Literature}

In this paper, a gait analysis method based on a magnetic angular rate and gravity sensor unit (MARG) was proposed. The sensor system monitors the subjects gait information using the estimated algorithm. Experimental results demonstrate that drift and error are well controlled by sensor fusion algorithm. Without pressure sensor, RFID tags and GPS, the self-contained MARG sensor based method can achieve an average distance error of $0.50 \%$ for level walking and an average height error of $1.7 \%$ for stair climbing in an indoor environment. It is difficult to directly compare the results with different experiments, because the algorithm performance is affected by many elements including the sensor type, the ground surface and the trajectory. Even though, our experimental results are comparable to or better than the literature:

1) Using the same type of MARG sensor, Ral et al. (2009) proposed a gyroscope based distance estimation method with $7.81 \%$ error, the averaged distance error is $1.27 \%$ in Jiménez Ruiz et al. (2012), the position error of $0.4 \%$ was reported in the study of Fourati (2015), which is quite accurate but lack of non-horizontal walking trials.

2) Using other types of sensor, the average distance estimation error for the indoor walking experiments is $5.5 \%$ with a standard deviation of $2.4 \%$ in the study of Yun et al. (2007), the distance error of $1.1 \%$ and the position error of $1.2 \%$ were reported in Godha and Lachapelle (2008), the distance error of $0.43 \%$ for short distance walking and $4.31 \%$ for long distance indoor walking were reported in Meng et al. (2014).

\section{Conclusion}

Since the orientation and position estimation merely needs a simple alignment and multiplications operations, this proposed method has the potential to provide real time tracking for various types of human activities. It is helpful for medical staff to evaluate gait information as the subject performs the predefined motions. Moreover, it can be used in many other applications that require real time bio-feedback. Hence it is a quite promising field to develop medical wearable device for ambulatory estimation of human activities.

Meanwhile, there exist some drawbacks in deploying wearable sensors. Most of wearable sensors are powered by batteries, which afford great flexibility but the limited battery capacity restricts the hours of use. This dilemma could be solved by more advanced battery technology. Furthermore, more sensor node are needed for wholebody motion monitor, then the energy and mobilityaware topology control must be addressed (Ghada et al., 2010). The other disadvantage of wearable sensors is the data packet dropout occurred in the process of wireless communication. This may be due to crosstalk, signal reflection or electromagnetic interference. A better wireless application protocol is helpful to reduce the loss of data. In the following research, we plan to evaluate various subjects including both healthy and pathological person at a wider movement range. In this way a complete training database can be set up.

\section{Acknowledgment}

This work was supported in part by National High Technology Research and Development Program (2012AA04150502), National Natural Science Foundation of China under Grant no.61174027, Liaoning Higher-education Outstanding Young Scholar Program (LJQ2012005) and the National Key Technology R\&D Program (2012BAK15B05).

Sen Qiu has been financially supported by scholarship from China Scholarship Council. The authors would like to express their thanks to these funding bodies.

\section{References}

Ai, H., Li, T., Wang, J., and Zhao, M. (2014). A new Wi-Fi fingerprint indoor localisation method for smart phone's heterogeneity. International Journal of Embedded Systems, 6(2/3):135 - 139.

Bachmann, E. R. and Zyda, M. J. (2000). Inertial and magnetic tracking of limb segment orientation for inserting humans into synthetic environments. Ph.D. dissertation, Naval Postgraduate School, Monterey, CA.

Bamberg, S. J. M., Benbasat, A. Y., Scarborough, D. M., Krebs, D. E., and Paradiso, J. a. (2008). Gait analysis using a shoe-integrated wireless sensor system. IEEE Transactions on Information Technology in Biomedicine, 12(4):413-23. 
Cavallo, F., a.M. Sabatini, and Genovese, V. (2005). A step toward GPS/INS personal navigation systems: real-time assessment of gait by foot inertial sensing. 2005 IEEE/RSJ International Conference on Intelligent Robots and Systems, pages 1187-1191.

Davis, R. B., Ounpuu, S., Tyburski, D., and Gage, J. R. (1991). A gait analysis data collection and reduction technique. Human Movement Science, 10:575-587.

Farago, I. and Geiser, J. (2007). Iterative operator-splitting methods for linear problems. International Journal of Computational Science and Engineering, 3(4):255 - 263.

Fourati, H. (2015). Heterogeneous Data Fusion Algorithm for Pedestrian Navigation via Foot-Mounted Inertial Measurement Unit and Complementary Filter. IEEE Transactions on Instrumentation and Measurement, 64(1):221 - 229 .

Ghada, K., Li, J., and Ji, Y. (2010). Energy and mobilityaware topology control in heterogeneous mobile ad hoc networks. International Journal of Computational Science and Engineering, 5(2):147 - 153.

Gilson, S. J., Fitzgibbon, A. W., and Glennerster, A. (2006). Quantitative analysis of accuracy of an inertial/acoustic 6DOF tracking system in motion. Journal of Neuroscience Methods, 154(1-2):175-82.

Godha, S. and Lachapelle, G. (2008). Foot mounted inertial system for pedestrian navigation. Measurement Science and Technology, 19(7):1-9.

Jiang, M., Shang, H., Wang, Z., Li, H., and Wang, Y. (2011). A method to deal with installation errors of wearable accelerometers for human activity recognition. Physiological Measurement, 32(3):347-58.

Jiménez Ruiz, A. R., Seco Granja, F., Prieto Honorato, J. C., and Guevara Rosas, J. I. (2012). Accurate pedestrian indoor navigation by tightly coupling foot-mounted IMU and RFID measurements. IEEE Trans.Instrum.Meas., 61(1):178-189.

Lamontagne, M., Beaulieu, M. L., and Beaulé, P. E. (2011). Comparison of joint mechanics of both lower limbs of THA patients with healthy participants during stair ascent and descent. Journal of Orthopaedic Research, 29(3):305-11.

Meng, X., Zhang, Z. Q., Wu, J. K., Wong, W. C., and Yu, H. (2014). Self-contained pedestrian tracking during normal walking using an inertial/magnetic sensor module. IEEE Transactions on Biomedical Engineering, 61(3):892-899.

Mihradi, S., Dirgantara, T., and Mahyuddin, A. I. (2011). Development of an Optical Motion-Capture System for 3D Gait Analysis. 2011 International Conference on Instrumentation, Communication, Information Technology and Biomedical Engineering, Indonesia, pages 391-394.

Nymark, J. R., Balmer, S. J., Melis, E. H., Edward, D., and Millar, S. (2005). Electromyographic and kinematic nondisabled gait differences at extremely slow overground and treadmill walking speeds. Journal of Rehabilitation Research and Development, 42(4):523534.

Qiu, S., Yang, Y., Hou, J., Ji, R., Hu, H., and Wang, Z. (2014). Ambulatory estimation of 3D walking trajectory and knee joint angle using MARG Sensors. Fourth International Conference on Innovative Computing Technology,Luton, UK (INTECH), pages $191-196$.
Ral, F., Eduardo, Z., and Jaime, G. (2009). Pedestrian tracking using inertial sensors. Journal of Physical Agents, 3(1):35-43.

Sabatini, A. M. (2012). Variable-State-Dimension Kalmanbased Filter for orientation determination using inertial and magnetic sensors. Sensors (Basel, Switzerland), 12(7):8491-506.

Skog, I., Händel, P., Nilsson, J.-O., and Rantakokko, J. (2010). Zero-velocity detection — an algorithm evaluation. IEEE transactions on bio-medical engineering, 57(11):2657-2666.

Srinivasan, S. and Ranganathan, H. (2013). RFID sensor network-based automation system for monitoring and tracking of sandalwood trees. International Journal of Computational Science and Engineering, 8(2):154-161.

Talpur, M. S. H., Bhuiyan, M. Z. A., and Wang, G. (2015). Shared-node IoT network architecture with ubiquitous homomorphic encryption for healthcare monitoring. International Journal of Embedded Systems, 7(1):43 - 54.

Wang, J., Lin, C., Yang, Y. C., and Ho, Y. (2012). Estimation Algorithms Using Gait Phase Information. IEEE Transactions on Biomedical Engineering, 59(10):28842892.

Wang, Z., Qiu, S., Cao, Z., and Jiang, M. (2013). Quantitative assessment of dual gait analysis based on inertial sensors with body sensor network. Sensor Review, 33(1):48-56.

Wang, Z. L., Zhao, H. Y., Qiu, S., and Gao, Q. (2015). Stance phase detection for ZUPT-aided footmounted pedestrian navigation system. IEEE/ASME Transactions on Mechatronics, (99).

Yun, X., Bachmann, E. R., Iv, H. M., and Calusdian, J. (2007). Self-contained Position Tracking of Human Movement Using Small Inertial/Magnetic Sensor Modules. IEEE International Conference on Robotics and Automation, Roma, Italy, pages 10-14. 